\title{
Patterns of Acute Non Amebiasis Dysentery in Adults
}

\author{
Azadeh Ebrahimzadeh, ${ }^{1}$ Bita Bijari, ${ }^{2,}{ }^{*}$ and Mohammad Hassan Namaei $^{3}$ \\ ${ }^{1}$ Hepatitis Research Center, Department of Infectious Diseases, Birjand University of Medical Sciences, Birjand, IR Iran \\ ${ }^{2}$ Hepatitis Research Center, Department of Community Medicine, Birjand University of Medical Sciences, Birjand, IR Iran \\ ${ }^{3}$ Hepatitis Research Center, Department of Microbiology, Birjand University of Medical Sciences, Birjand, IR Iran \\ "Corresponding author: Bita Bijari, Hepatitis Research Center, Department of Community Medicine, Birjand University of Medical Sciences, Birjand, IR Iran. E-mail: \\ bita.bijari@yahoo.com
}

Received 2015 May 15; Accepted 2015 September 06.

Keywords: Dysentery, Adults, Diarrhea

\section{Dear Editor,}

Diarrhea is still a major cause of death in many People all over the world. Acute diarrhea causes 3 million people death annually. Etiologic agents for bacterial dysentery are: Shigella (dysenteria, flexneri, boydii, sonnei), Salmonella (Typhi and para typhi), Campylobacter (jejuni) and Yersinia (entero colitica).

The most common agent in developing countries is Shigella dysenteria and in developed countries is Campylobacter jejuni [1]. While dysentery is a self limited disease, the antibiotic treatment not only reduces the symptomatic time, but also the excretion of the microbes, preventing its infection exposing other people [2]. The present study was aimed to determine patterns of acute non amebiasis dysentery in adult. This cross-sectional study performs in 2012. Patients with acute invasive diarrhea (bloody or mucoid diarrhea) for shorter than 3 days duration or watery diarrhea with fever or chill or tenesmus enrolled.

Exclusion criteria were lactating and pregnant women, antibiotic therapy in 72 hours past, patients with high toxicity and poor general condition and complications. In first, we get from all patients a sample of stool for direct examination and culture and antibiogram. Dysentery confirmed by positivity stool for WBC and RBC without amebiasis, then some of patients received azithromycin and other group received tab ciprofloxacin $500 \mathrm{mg} / 12$ hours for 5 days. After 48 hours, patients were examined for fever, general condition, decreased diarrhea and tenesmus. For patients with good clinical response, duration of treatment completed for 5 days but in groups without good clinical response, with use of results of culture and antibiogram, treatment changed. Stool culture was done from all patients in 5 th day after treatment. Also patients evaluate for drug reactions in two groups. Data was analyzed using SPSS-11 software and $\chi^{2}$ test was used $\mathrm{P}<0.05$ was set as the significant level.
From 84 patients, $52(61.9 \%)$ patients were male and 32 patients (38.9\%) were female. In 29 (34.5\%) patients culture result was negative. Shigella dysenteria cultured from $41(48.8 \%)$ patients and other organisms (E. coli, Salmonella paratyphi A, Campylobacter jejuni cultured in 14 (16.6\%) patients. Bloody diarrhea was seen at $38.1 \%$ patients and $27.3 \%$ had watery diarrhea and 32\% mucoid and mixed diarrhea. From 55 patients that had antibiogram, 32 (58.1\%) were sensitive to ciprofloxacin and $41(74.4 \%)$ to azithromycin. Clinical response in azithromycin was more than ciprofloxacin $93.5 \%$ versus $88.6 \%$. Stool cultures were negative in $100 \%$ patients at the end of treatment. In our study in patients with dysenteria, Shigella dysenteria is the most common etiologic agent but is not only bacterial etiology and other Gram negative bacillus example Salmonella paratyphi A, Campylobacter jejuni and E. coli. This may be to Shigella dysenteria low tolerance to hard environment conditions that it difficult Shigella dysenteria diagnosis or early antibiotic use by patients with dysenteria. Other studies showed quinolone resistance in Shigella (all species) has increased at an alarming speed, reinforcing the importance of continuous monitoring of antimicrobial resistance in Shigella (all species) [3]. In our study microbiological resistance for azithromycin was $19.3 \%$ and for ciprofloxacin was $23.8 \%$, that indicated Shigella dysenteria resistant to new antibiotics example macrolides and quinolones is increased but clinical response to azithromycin and ciprofloxacin were good.

\section{References}

1. Dupont HL. Shiglla species (bacillary dysentery). In: Mandell GL, Bennett JE, Dolin R, editors. Mandell, Douglas, and Bennett's principles and practice of infectious diseases. Philadelphia: Churchill Livingstone; 2010. pp. 2905-10.

2. Jain SK, Gupta A, Glanz B, Dick J, Siberry GK. Antimicrobial-resistant Shigella sonnei: limited antimicrobial treatment options for children 
and challenges of interpreting in vitro azithromycin susceptibility. Pediatr Infect Dis J. 2005;24(6):494-7. [PubMed: 15933557].

3. Gu B, Cao Y, Pan S, Zhuang L, Yu R, Peng Z, et al. Comparison of the prevalence and changing resistance to nalidixic acid and ciprofloxacin of Shigella between Europe-America and Asia-Africa from 1998 to 2009. Int J Antimicrob Agents. 2012;40(1):9-17. doi: 10.1016/j.ijantimicag.2012.02.005. [PubMed: 22483324]. 